\title{
Avanços na Síntese Química: Síntese Assistida por Micro-Ondas
}

\author{
Ana M. G. Silva ${ }^{1, *}$, Vera L. M. Silva ${ }^{2,},{ }^{*}$ Carla Queirós ${ }^{1}$, Joana Pinto ${ }^{2}$
}

\begin{abstract}
0
uso da radiação de micro-ondas como fonte de energia para a realização de transformações químicas tem sido extensivamente investigado durante os últimos anos, mas também tem levantado alguma controvérsia. Assim, este artigo tem por objetivo mostrar a crescente importância do aquecimento usando radiação micro-ondas em síntese química, dando especial ênfase a trabalhos desenvolvidos em Portugal, bem como às vantagens e limitações inerentes a esta técnica. Como existem vários instrumentos de micro-ondas para uso à escala laboratorial disponíveis comercialmente e com diferentes características, este artigo irá focar-se nos diferentes instrumentos existentes e em alguns resultados obtidos em cada um deles. Reações em paralelo assistidas por radiação micro-ondas, bem como o seu escalamento serão também abordadas e ilustradas através de exemplos.
\end{abstract}

\section{INTRODUÇÃO}

A irradiação por micro-ondas como método de aquecimento foi originalmente observada em 1946 por Spencer quando realizava experiências no seu laboratório com vista à obtenção de um novo tubo de vácuo chamado magnetrão [1]. Numa dessas experiências, ele descobriu acidentalmente que uma barra de chocolate que tinha no bolso derreteu após exposição à radiação micro-ondas. Spencer desenvolveu a ideia e rapidamente concluiu que a radiação micro-ondas poderia ser usada como método de aquecimento. Logo no ano seguinte, em 1947, Spencer projetava o primeiro forno de micro-ondas para uso doméstico [1]. Desde então, o desenvolvimento da radiação micro-ondas como fonte de aquecimento tem sido muito gradual, tal como mostra a Figura 1.

Só quatro décadas mais tarde é que se realizaram os primeiros ensaios de síntese química assistida por radiação micro-ondas. Foi em 1986 que Gedye e Giguere descreveram as primeiras reações de síntese orgânica em vasos abertos envolvendo radiação micro-ondas, utilizando para isso fornos de micro-ondas domésticos, sem qualquer controlo de temperatura ou

\footnotetext{
${ }^{2}$ REQUIMTE, Departamento de Química, Faculdade de Ciências, Universidade do Porto, 4169-007 Porto

Departamento de Química \& QOPNA, Universidade de Aveiro, 3810-193 Aveiro

*E-mail: ana.silva@fc.up.pt; verasilva@ua.pt
}

pressão [2, 3]. Apesar de muitas explosões violentas resultantes do rápido e descontrolado aquecimento de solventes orgânicos, os químicos de síntese cedo perceberam que esta nova tecnologia viria a trazer grandes vantagens em relação às técnicas convencionais de aquecimento. Em alguns casos, o aquecimento usando radiação micro-ondas permitiu realizar reações muito mais rapidamente do que em condições de aquecimento convencionais.

Na década de 90 começaram a surgir os primeiros fornos micro-ondas para uso em laboratório. Em 1990, Milestone S. R. L. desenvolveu o vaso HPV 80 que permitiu a digestão completa a alta pressão de materiais como óxidos, óleos e compostos farmacêuticos considerados de difícil digestão. Entre 1992 e 1996, a CEM Corporation introduziu um novo sistema, o MDS 2000, que possibilitou aumentar o número de ensaios executados em simultâneo na digestão com radiação micro-ondas. Durante o mesmo período, a CEM Corporation introduziu também um sistema de cavidade monomodo ou mono simples (Star 2), que veio permitir um melhor controlo das condições de digestão [4].

Desde então, esta tem sido uma das áreas mais desenvolvidas da química orgânica, o que é evidenciado pelo crescente número de artigos publicados sobre este tópico $[5,6]$. Uma pesquisa bibliográfica utilizando as palavras-chave "microwave" e "organic synthesis" deu origem a mais de 900 artigos científicos publicados em jornais nos últimos cinco anos (20052010), mais do que o dobro do número de artigos publicados nos cinco anos anteriores (2000-2005) [7]. Para além da química orgânica, a síntese assistida por radiação micro-ondas tem sido utilizada com grande sucesso noutras áreas da química, das quais se destacam a química inorgânica, química dos polímeros e materiais, nanotecnologias e processos bioquímicos. $\mathrm{Na}$ grande maioria dos casos, o aquecimento usando radiação micro-ondas

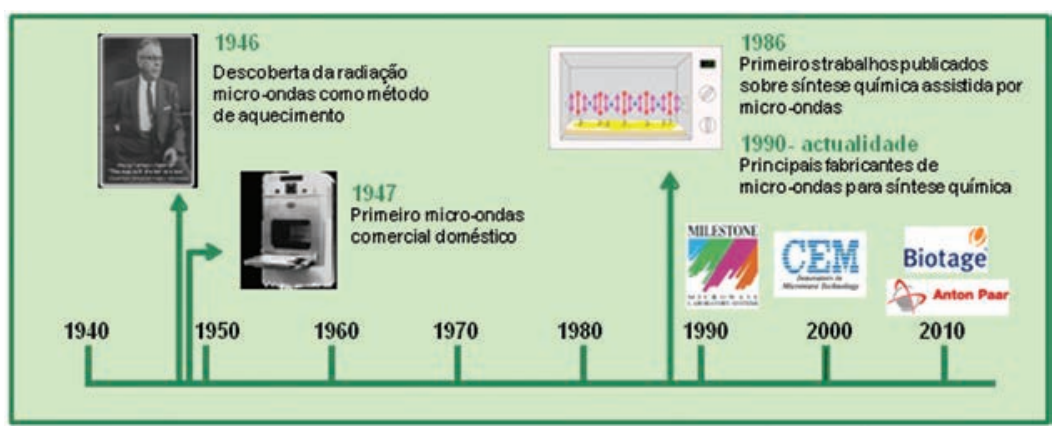

Figura 1 - Evolução do aquecimento por micro-ondas 
permite reduzir drasticamente os tempos e aumentar o rendimento das reações.

Curiosamente, em Portugal, nos últimos 10 anos (2000-2010) registaram-se 65 artigos em diferentes áreas da química, destacando-se a química orgânica, a química inorgânica, a síntese de materiais híbridos orgânicos-inorgânicos (Metal-Organic Frameworks, MOFs) e nanomateriais. Tal como mostra o Gráfico 1, os trabalhos de síntese só tiveram início em 2002 e em 2008 ocorreu o maior número de publicações de trabalhos nas áreas mencionadas anteriormente.

\section{PrincíPIOS dO AQUECIMENTO USANDO RADIAÇÃO MICRO-ONDAS}

Tradicionalmente, as reações químicas são aquecidas com recurso a uma fonte de calor externa, manta de aquecimento ou banho de óleo. Esta forma de aquecimento é um método bastante lento e ineficiente de transferência de energia para uma mistura reacional, pois apresenta uma elevada inércia térmica, uma vez que depende de processos de convecção e condução do calor e da condutividade térmica dos diferentes materiais através dos quais o calor tem de penetrar, o que resulta muitas vezes numa temperatura do vaso de reação superior à da mistura reacional.

Por sua vez, o aquecimento usando radiação micro-ondas proporciona um aquecimento mais eficiente, já que este é gerado internamente por absorção direta da radiação micro-ondas por moléculas polares presentes na mistura reacional (por exemplo solventes, reagentes e catalisadores). $\mathrm{Na}$ Tabela 1 apresentam-se as características principais do aquecimento convencional versus aquecimento com radiação micro-ondas.

A radiação micro-ondas $(2,45 \mathrm{GHz}$ é a frequência utilizada nos fornos domésticos e de síntese química) é convertida em aquecimento segundo dois mecanismos principais: polarização dipolar e condução iónica. Os iões ou dipolos presentes numa dada reação, quando sujeitos à radiação micro-ondas, vão alinhar-se segundo o campo

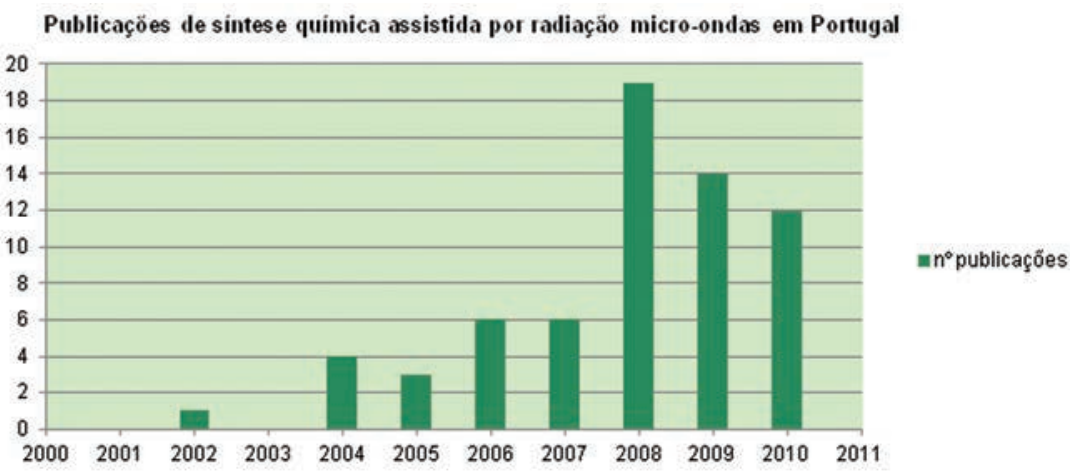

Gráfico 1 - Número de publicações de síntese química assistida por micro-ondas em Portugal na última década (2000-2010)

Tabela 1 - Aquecimento convencional versus aquecimento com radiação micro-ondas

Aquecimento convencional
$\begin{gathered}\text { A energia é transferida através das paredes } \\ \text { do vaso reacional e, em seguida, dissipada } \\ \text { para a mistura reacional }\end{gathered}$
$\begin{gathered}\text { A radiação micro-ondas é absorvida } \\ \text { diretamente pelas moléculas presentes na } \\ \text { mistura reacional, sendo as paredes do vaso } \\ \text { reacional transparentes à radiação }\end{gathered}$

elétrico aplicado. Como o campo aplicado oscila, os iões ou dipolos vão realinhar-se com a corrente elétrica alternada e, durante esse processo, a energia é perdida na forma de calor através de fricção molecular e perdas dielétricas. A quantidade de calor gerado por este processo está diretamente relacionada com a capacidade dos materiais para se alinharem à frequência do campo aplicado. Se o ião ou dipolo não tem tempo para realinhar ou reorienta muito rapidamente com o campo aplicado, então não há aquecimento. Deste modo, quando se planifica uma experiência com radiação micro-ondas deve ter-se em conta as propriedades dielétricas do material a aquecer

A capacidade de uma dada substância converter energia eletromagnética em calor é determinada por tan $\delta$, grandeza que está relacionada com a constante dielétrica $(\varepsilon)$ do material. Uma substância com elevada cons- tante dielétrica irá absorver radiação com elevada eficiência e, consequentemente, aquecer mais rapidamente. Geralmente, os solventes mais polares como álcoois, ácidos e DMSO, têm valores elevados de constante dielétrica, pelo que são considerados bons solventes para usar com radiação micro-ondas, enquanto os solventes apolares, como hexano e tolueno, possuem constantes dielétricas muito baixas e, por isso, são considerados transparentes à radiação (Tabela 2 ).

Os reagentes e catalisadores da reação também contribuem para as propriedades dielétricas globais da mistura reacional. Por exemplo, os líquidos iónicos podem ser adicionados a uma mistura reacional só com o intuito de aumentar a sua capacidade de absorção da radiação $[8,9]$. Aditivos como o carboneto de silício (SiC) podem ser também adicionados a solventes apolares de modo a melhorar a absorção de energia pela mistura reacional e, 
consequentemente, aumentar a sua velocidade de aquecimento. Os elementos de $\mathrm{SiC}$ são térmica e quimicamente resistentes a temperaturas até $1500^{\circ} \mathrm{C}$ e compatíveis com qualquer solvente ou reagente [10].

\section{INSTRUMENTAÇÃO}

Embora operacionalmente simples e relativamente baratos, os aparelhos de micro-ondas domésticos utilizados nas primeiras experiências de síntese orgânica sofreram com as vicissitudes próprias deste tipo de equipamento (distribuição de carga irregular, pontos quentes, etc.), e com as limitações técnicas (falta de controlo de temperatura, perda descontrolada de componentes voláteis, etc.), estando estas estritamente relacionadas com a falta de segurança dos equipamentos. Todas estas limitações, associadas à falta de reprodutibilidade dos protocolos realizados, levaram os fabricantes de aparelhos de micro-ondas a procurarem construir instrumentos mais adequados à síntese química, que incluem dispositivos de controlo de temperatura (sensores de infravermelho, termopares e fibra-ótica) e de pressão nos vasos reacionais, e dispositivos de segurança adequados à realização de transformações químicas. Assim, surgiram os equipamentos específicos para síntese assistida por radiação micro-ondas. Atualmente, existem no mercado quatro fabricantes principais que desenvolvem e comercializam os instrumentos de micro-ondas para síntese química: Biotage, CEM, Milestone e Anton Paar.

Relativamente ao desenho do reator, este pode ser do tipo multimodo ou monomodo. Nos reatores do tipo multimodo, a radiação micro-ondas é refletida nas paredes da cavidade larga do forno e um suporte rotativo permite que a distribuição do campo seja o mais homogénea possível. Em contraste, nos reatores monomodo ou modo simples, a radiação eletromagnética é focada diretamente no reator montado a uma distância fixa da fonte de radiação (Tabela 3 ).

A diferença fundamental entre estes dois tipos de sistemas de reatores reside no facto de que em cavidades multimodo se podem irradiar vários

Tabela 2 - Valores de $\tan \delta$ de alguns solventes usados em síntese

\begin{tabular}{|c|c|c|c|}
\hline solvente & $\tan \delta$ & solvente & $\tan \delta$ \\
\hline etilenoglicol & 1,350 & DMF & 0,161 \\
\hline etanol & 0,941 & 1,2-dicloroetano & 0,127 \\
\hline DMSO & 0,825 & água & 0,123 \\
\hline 2-propanol & 0,799 & clorobenzeno & 0,101 \\
\hline ácido fórmico & 0,722 & clorofórmio & 0,091 \\
\hline metanol & 0,659 & acetonitrilo & 0,062 \\
\hline nitrobenzeno & 0,589 & acetato de etilo & 0,059 \\
\hline 1-butanol & 0,571 & acetona & 0,054 \\
\hline 2-butanol & 0,447 & tetra-hidrofurano & 0,047 \\
\hline 1,2-diclorobenzeno & 0,280 & diclorometano & 0,042 \\
\hline 1-metil-2-pirrolidinona & 0,275 & tolueno & 0,040 \\
\hline ácido acético & 0,174 & hexano & 0,020 \\
\hline
\end{tabular}

Tabela 3 - Cavidade monomodo versus cavidade multimodo

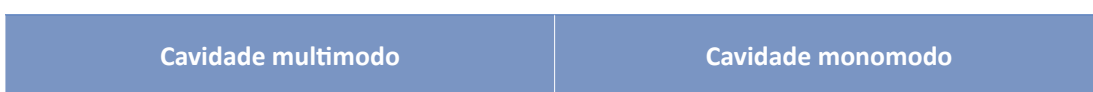

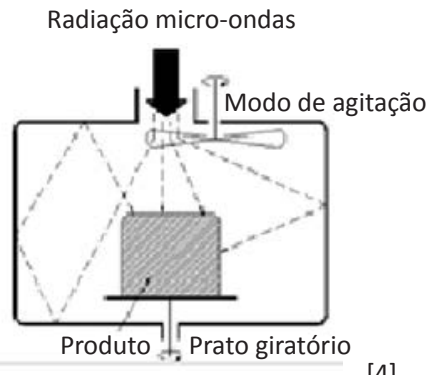

$[4]$

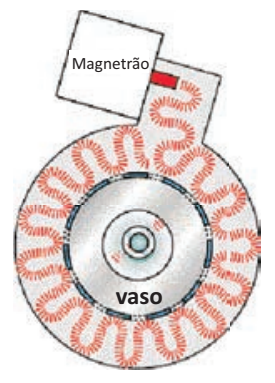

[11]

\begin{tabular}{cc}
\hline $\begin{array}{c}\text { A radiação micro-ondas é refletida } \\
\text { nas paredes da cavidade do forno }\end{array}$ & $\begin{array}{c}\text { A radiação micro-ondas é direcionada } \\
\text { ao vaso reacional }\end{array}$ \\
\hline Energia dispersa na cavidade & Aquecimento mais homogéneo \\
\hline $\begin{array}{c}\text { Podem ser irradiados vários vasos } \\
\text { simultaneamente }\end{array}$ & Só pode ser irradiado um vaso de cada vez \\
\hline
\end{tabular}

vasos reacionais simultaneamente em suporte rotativo (síntese paralela), enquanto que nos sistemas monomodo só se pode irradiar um vaso de cada vez. Além disso, a potência disponível em sistemas multimodo (em geral de 1000 a 1400 W) é superior à potência disponível em sistemas monomodo (em geral de 300 a $850 \mathrm{~W}$ ).

Para além das características técnicas específicas de cada marca e de cada tipo de reator, há ainda outras questões importantes que têm que ser abordadas aquando da realização duma síntese usando radiação micro-ondas. Por exemplo, as reações na ausência de solvente foram já muito populares no meio científico, no entanto, dificuldades técnicas relacionadas com o aquecimento não uniforme, com a mistura dos componentes da reação e com a monitorização da temperatura permanecem 
sem resposta, o que conduziu, de certo modo, ao seu abandono. Deste modo, privilegia-se a síntese assistida por radiação micro-ondas em solução, podendo esta ser realizada em vaso aberto ou vaso fechado. Quando se trabalha em solução e em condições de vaso aberto, os solventes são aquecidos por absorção da radiação micro-ondas à pressão atmosférica. Nestas condições, o ponto de ebulição do solvente limita a temperatura de reação. Assim, com intuito de se alcançarem rendimentos mais elevados, são frequentemente utilizados em vaso aberto solventes com boa absorção de radiação micro-ondas e alto ponto de ebulição. No entanto, o uso desses solventes (por exemplo, DMF, 1-metil-2-pirrolidinona e etilenoglicol) apresenta sérios desafios durante o tratamento e purificação do produto da reação. Em resposta a estas limitações surgiram então reatores de micro-ondas mais modernos, com monitorização online da temperatura e pressão, para a realização de reações em vaso fechado. Nestas condições, podem ser rapidamente atingidas temperaturas acima do ponto de ebulição do solvente. É a combinação do aquecimento rápido usando radiação micro-ondas com a tecnologia de vaso fechado que tem sido responsável pelos bons resultados observados na síntese química.

Kappe e o seu grupo de investigação da Universidade de Graz procuram dar resposta a uma das questões mais controversas em torno do aquecimento com radiação micro-ondas: Existe ou não efeito não térmico no aquecimento com radiação micro-ondas? Estes investigadores utilizam vasos de reação de SiC para separar os efeitos térmicos da radiação, da própria radiação. O SiC é ideal para este fim já que absorve na totalidade a radiação micro-ondas, o que faz com que o vaso reacional aqueça durante esse processo e transfira o calor para o meio reacional. Segundo Kappe, esta característica dos vasos de SiC permite utilizar solventes não polares de pouca absorção, como é o caso do tolueno ou hexano, que normalmente não se conseguiam aquecer em aparelhos de micro-ondas, mas permite também o uso de bases muito corrosivas, como hidróxidos e reagentes como o HF, que normalmente atacam o vidro. Permite ainda a realização de transformações que envolvam o uso de metais, como por exemplo reações de Grignard [12]. A comparação de experiências envolvendo o uso de vasos de $\mathrm{SiC}$ com os vasos tradicionais de borosilicato sugerem que existem apenas efeitos térmicos, não havendo efeitos não térmicos resultantes do campo eletromagnético da radiação micro-ondas [12].

De facto, esta tecnologia avançada de aquecimento está em constante evolução. Recentemente, as técnicas de fluxo contínuo têm vindo a ganhar uma importância crescente, principalmente no que diz respeito ao escalamento para maiores quantidades (instalação piloto ou industrial). Nesta técnica, a mistura reacional é passada através de uma célula de fluxo transparente posicionada dentro de um reator de micro-ondas apropriado. Em fluxo contínuo é necessário ter em conta o tempo de reação optimizador para a reação em batch, e relacioná-lo com o tempo de permanência no reator (o tempo que a mistura reacional permanece na célula de aquecimento por radiação micro-ondas) a uma razão volume/caudal específicos. Os recentes avanços em processamento de fluxo contínuo permitem já realizar, entre outras transformações, a síntese da aspirina num só passo em mistura homogénea em reatores comerciais adaptados para trabalhar em fluxo contínuo [13-15].

Paralelamente têm surgido novos reatores em batch para trabalhar à escala do quilograma [16-18]. Recentemente, uma série de reações de síntese de compostos com elevada importância farmacêutica foram realizadas à escala do quilograma utilizando o reator comercial Synthos 3000 em batch [18]. Este reator permite trabalhar com volumes relativamente grandes de mistura reacional (máximo de 16 vasos com capacidade de $70 \mathrm{~mL}$ cada) utilizando temperaturas e pressões altas $\left(240^{\circ} \mathrm{C} / 40\right.$ bar $)$. Os resultados mostram reduções significativas nos tempos das reações e bons rendimentos. No entanto, este equipamento apresenta ainda algumas limitações relacionadas com a agitação magnética pouco eficiente, o tempo de arrefecimento muito longo e o facto de não ser possível realizar vários batches sequencialmente de forma automática.

Existem ainda aparelhos de micro-ondas hifenados que incluem, por exemplo, a combinação da radiação micro-ondas com ultrassons, bem como com radiação ultravioleta [19].

Assim podemos concluir que, no que respeita ao escalamento das reações assistidas por radiação micro-ondas, ainda não existe nenhuma tecnologia disponível comercialmente que possa ser utilizada em todas e quaisquer circunstâncias, sem apresentar um conjunto de limitações.

\section{VANTAGENS DA SÍNTESE ASSISTIDA POR RADIAÇÃO MICRO-ONDAS}

As vantagens desta tecnologia têm sido demonstradas em várias áreas da química, desde o desenvolvimento de metodologias sintéticas até à aplicação em processos industriais [20].

As principais vantagens associadas ao aquecimento com radiação micro-ondas são:

- Obtenção de temperaturas de reação elevadas através da combinação da radiação micro-ondas comautilização devasosfechados.

- Redução dos tempos de reação, rendimentos elevados e reações mais "limpas", facilitando os processos de purificação.

- Utilização de solventes de baixo ponto de ebulição em vasos fechados sob pressão e a temperaturas elevadas (acima do ponto de ebulição do solvente).

- Combinação da radiação microondas com catalisadores que absorvem fortemente a radiação.

- Monitorização online da temperatura e pressão estabelecendo condições experimentais mais facilmente reprodutíveis.

- Adaptação a síntese sequencial automatizada ou síntese paralela.

- Possibilidade de combinação com outras técnicas, como por exemplo sonoquímica e fotoquímica. 
Desvantagens dA SÍNTESE ASSISTIDA POR RADIAÇÃO MICRO-ONDAS

A utilização laboratorial em síntese orgânica de aparelhos de micro-ondas em substituição dos métodos de aquecimento convencionais (banhos de óleo e mantas de aquecimento) tem-se generalizado e, além disso, tem sido apresentada como sendo um processo energeticamente eficiente. Mas será o aquecimento usando radiação micro-ondas um processo de aquecimento energeticamente eficiente? A resposta a esta questão é difícil por dois motivos, só existem relatos sobre este assunto a partir de 2005 e são poucos. Por outro lado, o cálculo do consumo de energia para uma reação aquecida usando radiação micro-ondas é complexo [21-23]. As principais desvantagens e limitações associadas a esta tecnologia são:

- A baixa eficiência do magnetrão, dado que apenas $50 \%$ da energia elétrica usada é transformada em energia micro-ondas. Alguns estudos demonstram que, no caso de refluxo em vaso aberto, o aquecimento com radiação micro-ondas consome significativamente mais energia do que as técnicas de aquecimento convencionais [24, 25].

- Nem toda a radiação gerada é absorvida pela mistura reacional. Alguns estudos demonstram que os consumos de energia no aquecimento com radiação micro-ondas dependem da natureza do solvente, mais precisamente do seu momento dipolar, que afeta as características de absorção da radiação pela mistura reacional, e do seu ponto de ebulição, bem como da escala de operações - à escala laboratorial $(5-200 \mathrm{mmol}$ de reagentes num volume de 4-300 ml) [5].

- O aquecimento com radiação micro-ondas pode não ser exequível, por exemplo se a reação requer um solvente apolar cuja absorção da radiação é limitada. Este problema pode ser contornado usando reagentes, catalisadores, etc., que sejam polares e proporcionem a absorção, ou então usando um agente passivo, fortemente absor- vente (por exemplo uma vareta de $\mathrm{SiC}$ inserida no reator), que facilite o aquecimento [26].

- Embora a energia que é necessária para aquecer um tubo reacional selado num reator monomodo seja pequena, não se pode estabelecer uma comparação direta com o banho de óleo no qual é possível aquecer volumes maiores ou então vários tubos ao mesmo tempo. Quando comparado com mantas de aquecimento elétricas, onde a maioria da energia elétrica é convertida em calor, o aquecimento com radiação micro-ondas é pouco eficiente. Este tipo de aquecimento também se revela pouco eficiente quando são aquecidas reações à pressão atmosférica, comparativamente com os resultados obtidos para reações em vaso aberto aquecidas de forma convencional.

- O escalamento para a grande escala industrial é bastante problemático e difícil de realizar, devido à profundidade de penetração da radiação micro-ondas em meios absorventes ser limitada, além de que a questão da segurança também levanta problemas. Além disso, o aquecimento homogéneo em grande escala não é garantido, não se conseguindo saber o valor exato da temperatura a que se está a operar. Até ao momento, o escalamento foi realizado apenas à escala laboratorial, da ordem de algumas gramas para centenas de gramas ou, em reatores de fluxo, e mais recentemente em reatores em batch, para a escala intermédia de alguns quilogramas [27, 28].

- Os reatores de fluxo são naturalmente abertos, pelo que também não possibilitam o aquecimento em vaso selado. Deste modo, o escalamento de situações em que o uso de radiação micro-ondas teve mais êxito no laboratório parece problemático e o escalamento para maiores quantidades (instalação piloto ou industrial) só poderá ser realizado por replicação de reatores [29].

- Muitas aplicações industriais que são realizadas rotineiramente e em larga escala são efetuadas em reatores especialmente projetados, normalmente com guias de onda otimizados e muitas vezes em frequências alternativas (já que isso também afeta a eficiência da transferência de energia). Contudo, a adaptação de reatores a cada caso torna-os obviamente menos versáteis, mais limitados e com custos mais elevados.

- O controlo da temperatura depende fortemente do método de medição, assim como da posição do dispositivo de medição no reator. Isso torna difícil a comparação com as condições de reação convencionais e leva a especulações sobre a existência de "efeitos não térmicos" nas reações químicas assistidas por micro-ondas [30].

- A tecnologia envolvida na conceção dos aparelhos de micro-ondas faz com que o elevado custo de investimento seja mais uma desvantagem.

Resumindo, o aquecimento com radiação micro-ondas possui várias limitações e nem sempre possibilita um aumento da eficiência relativamente aos aquecimentos convencionais, pelo que a eficiência energética deverá ser avaliada caso a caso.

\section{SÍNTESE ASSISTIDA POR RADIAÇÃO MICRO- -ONDAS EM PORTUGal}

Ao contrário do que aconteceu noutros países, em que os primeiros trabalhos foram realizados em vaso aberto utilizando equipamentos de micro-ondas domésticos, em Portugal, a investigação começou já com a utilização de equipamento específico para síntese.

Uma das primeiras áreas a serem exploradas foi a química orgânica refere-se como exemplo a utilização da radiação micro-ondas na síntese de 3-estirilcromonas através da condensação de 3-formilcromonas com ácidos fenilacéticos [31]. Outro exemplo que ilustra bem a importância do aquecimento com radiação micro-ondas em síntese orgânica envolve a preparação de porfirinas fundidas ao anel piridinona a partir de reações de ciclização. Foram testados três protocolos: aquecimento convencional, aquecimento com radiação micro-ondas e reagente de Eaton (mistura de $\mathrm{P}_{2} \mathrm{O}_{5}$ e $\mathrm{MeSO}_{3} \mathrm{H}$ ) [32]. Na realização das reações por aquecimento com ra- 
diação micro-ondas foram utilizados dois reatores diferentes: multimodo e monomodo. Os resultados mostram que no reator multimodo, em condições de vaso aberto, a ciclização foi completa após $40 \mathrm{~min}$ a $180^{\circ} \mathrm{C}$ (temperatura de ebulição do solvente). Já com o reator monomodo, em vaso fechado, e aumentando a temperatura da reação para $220^{\circ} \mathrm{C}$, a ciclização completou-se em apenas $4 \mathrm{~min}$, com uma notável redução no tempo de reação (Tabela 4). Já em condições convencionais (entrada 1) e com o reagente de Eaton (entrada 4), a ciclização é muito mais lenta ( 6 h e 2 h, respetivamente), obtendo-se rendimentos relativamente mais baixos.

Os compostos do tipo estirilpirazóis (Figura 2) são muito relutantes a participar como dienos em reações de Diels-Alder envolvendo o anel do pirazol devido à perda de aromaticidade inerente a estas reações. Por isso as reações de cicloadição de estirilpirazóis requerem dienófilos muito reativos, assim como temperaturas e pressões elevadas $\left(120-140^{\circ} \mathrm{C}\right.$ e 8-10 atm), e geralmente são reações lentas, dando origem aos correspondentes cicloaductos em rendimentos moderados. No entanto, através da reação de estirilpirazóis com $\mathrm{N}$-metilmaleimida em aquecimento com micro-ondas, num reator multimodo, sem solvente, à pressão atmosférica e a uma potência de 800 W, foi possível obter os cicloadutos com bons rendimentos [33].

Um outro exemplo, bastante ilustrativo, refere-se à síntese de 4-isoxazolinas (Figura 2) via reações de cicloadição 1,3-dipolares de nitronas geradas a partir de alenoatos, seguidas de rearranjo térmico para dar origem a pirróis. Neste caso verificou-se que a principal vantagem da aplicação da radiação micro-ondas está relacionada com a diminuição do tempo da reação [34]. Ainda no contexto da utilização da radiação micro-ondas em reações de cicloadição 1,3-dipolares, foi recentemente publicado um artigo de revisão que destaca os desenvolvimentos mais recentes nesta área, com especial ênfase para aspetos relacionados com a régio- e estereosselectividade e eficiência das reações [35].

Tabela 4 - Protocolos de Ciclização

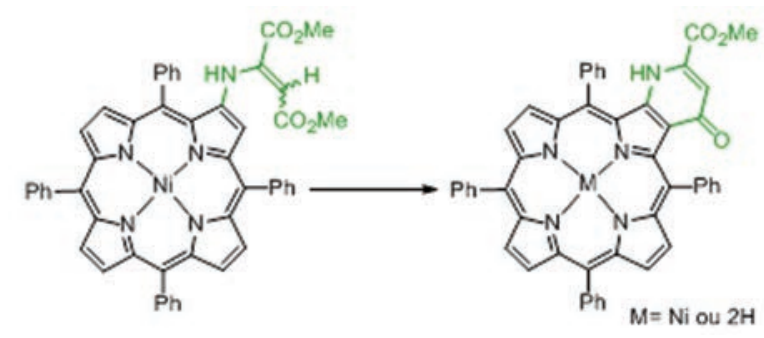

\begin{tabular}{|c|c|c|c|c|}
\hline Entrada & Método & Temp $\left({ }^{\circ} \mathrm{C}\right)$ & Tempo & Rendimento (\%) \\
\hline 1 & Banho de óleo & 200 & $6 \mathrm{~h}$ & 74 \\
\hline $2 *$ & Micro-ondas vaso aberto & 180 & $40 \mathrm{~min}$ & 93 \\
\hline $3 * *$ & Micro-ondas vaso fechado & 220 & $4 \min$ & 88 \\
\hline 4 & Reagente de Eaton & 50 & $2 \mathrm{~h}$ & 84 \\
\hline
\end{tabular}<smiles>[R]c1ccc(/C=C/c2cn(C(C)=O)nc2-c2ccccc2O)cc1</smiles>

estirilpirazóis<smiles>[R]CC1(CC(=O)OCc2ccccc2)C([R])=C([R])ON1[R2]</smiles>

4-isoxazolinas

Figura 2 - Estruturas de estirilpirazóis e 4-isoxazolinas

A catálise assistida por radiação micro-ondas também tem sido explorada com sucesso. Exemplo disso é o trabalho desenvolvido por Pombeiro e colaboradores que utilizam complexos de alcoxi-triazapentadienato cobre(II) como catalisadores muito eficientes em reações de oxidação assistida por radiação micro-ondas de álcoois secundários em cetonas, na presença de $t$-butil-hidroperóxido e na ausência de solvente (ver Tabela 5) [36]. Nestas condições (30 minutos, $80^{\circ} \mathrm{C}$, usando a radiação micro-ondas), o 1-feniletanol é oxidado a acetofenona com rendimento quantitativo enquanto, nas mesmas condições, mas utilizando aquecimento convencional, o produto é apenas obtido com rendimento de $4 \%$.

A epoxidação de olefinas, tais como cis-cicloocteno e R-(+)-limoneno, utilizando complexos de molibdénio como catalisadores na presença de t-butil-hidroperóxido, foi também explorada com recurso ao aquecimento convencional e à catálise assistida por micro-ondas [37, 38]. Os resultados demonstraram que estes dois métodos de aquecimento não afetam a seletividade das reações, mas afetam significativamente a sua velocidade (geralmente em micro-ondas é necessário menos tempo para atingir a mesma percentagem de conversão).

Mais recentemente, o aquecimento com radiação micro-ondas tem despertado a atenção de outras áreas, nomeadamente de ciências dos materiais. O interesse na avaliação de parâmetros como a qualidade dos cristais, a sua morfologia e tamanho tem levado muitos investigadores a reproduzirem sínteses de MOFs já conhecidas com radiação micro-ondas ou a desenvolverem novos materiais usando radiação micro-ondas para 
diferentes aplicações. Rocha e colaboradores, durante a realização de experiências com vista à obtenção de sistemas lantanídeo/ ácido 2,5-piridinodicarboxílico usando o aquecimento com radiação micro-ondas, observaram inesperadamente a síntese de uma nova estrutura de MOF com canais largos unidimensionais. Este material foi isolado em condições suaves em apenas 30 minutos de reação [39]. Os desenvolvimentos mais significativos da síntese de MOFs assistida por radiação micro-ondas foram muito recentemente revistos pelos mesmos autores, enfatizando as principais vantagens deste método de aquecimento no isolamento de grandes quantidades desses materiais em períodos de tempo mais curtos [40].

\section{SíNTESE ASSISTIDA POR RADIAÇÃO MICRO-ONDAS: QUE FUTURO?}

O aquecimento por micro-ondas é uma técnica ainda muito recente, com uma margem de aperfeiçoamento maior do que outras técnicas. Apesar da intensa investigação desenvolvida ao longo destes anos, existem ainda muitas limitações que não foram ultrapassadas. Uma das maiores limitações está relacionada com o alto custo da energia de micro-ondas por quilowatt instalado e a relativa ineficiência do magnetrão na conversão de energia elétrica em energia micro-ondas. Atualmente, os fabricantes de aparelhos de micro-ondas continuam a desenvolver esforços com vista à construção de magnetrões mais eficientes e aperfeiçoamento dos de- senhos dos reatores já existentes, principalmente no que diz respeito a questões de segurança. Deste modo, espera-se o aparecimento de novos reatores de micro-ondas, energeticamente mais eficientes, capazes de trabalhar em condições de elevada pressão e temperatura e em larga escala.

\section{Agradecimentos}

Os autores agradecem ao projeto PTDC/QUI-QUI/102454/2008, financiado pela Fundação para a Ciência e a Tecnologia (FCT).

\section{REFERÊNCIAS}

[1] J.M. Osepchuk, IEEE MTT-S International Microwave Symposium Digest 5165967 (2009) 1397.

[2] R. Gedye, F. Smith, K. Westaway, H. Ali, L. Baldisera, L. Laberge, J. Rousell, Tetrahedron Lett. 27 (1986) 279.

[3] R.J. Giguere, T.L. Bray, S.M. Duncan, G. Majetich, Tetrahedron Lett. 27 (1986) 4945.

[4] M. Taylor, Developments in Microwave Chemistry, Evalueserve, RSC, 2005.

[5] C.O. Kappe, Angew. Chem. Int. Ed. 43 (2004) 6250.

[6] C.O. Kappe, D. Dallinger, Mol. Divers. 13 (2009) 71.

[7] Scopus data.

[8] N.E. Leadbeater, H.M. Torenius J. Org. Chem. 67 (2002) 3145.

[9] E. Van der Eycken, P. Appukkuttan, W. De Borggraeve, W. Dehaen, D. Dallinger, C.O. Kappe J. Org. Chem. 67 (2002) 7904.

Tabela 5 - Exemplo de catálise assistida por micro-ondas [36]

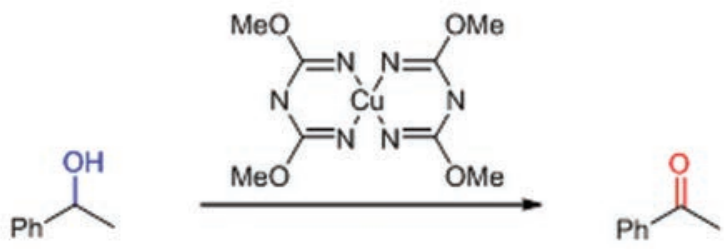

\begin{tabular}{cccc}
\hline Método & Temp $\left({ }^{\circ} \mathrm{C}\right)$ & Tempo & Rendimento (\%) $^{a}$ \\
\hline $\begin{array}{c}\text { Aquecimento convencional } \\
\text { Aquecimento usando radiação } \\
\text { micro-ondas }\end{array}$ & 80 & $30 \mathrm{~min}$ & 4 \\
\hline \begin{tabular}{c} 
a Valores obtidos no GC \\
\hline
\end{tabular}
\end{tabular}

[10] J.M. Kremsner, C.O. Kappe, J. Org. Chem. 71 (2006) 4651.

[11] Self-turning Patented Focused ${ }^{\mathrm{TM}}$ single-mode cavity system by CEM Corporation.

[12] D. Obermayer, B. Gutmann, C.O. Kappe, Angew. Chem. Int. Ed. 48 (2009) 8321.

[13] M.H.C.L. Dressen, B.H.P. van de Kruijs, J. Meuldijk, J.A.J.M. Vekemans, L.A. Hulshof, Org. Process Res.Dev. 14 (2010) 351.

[14] M.D. Bowman, J.L. Holcomb, C.M. Kormos, N.E. Leadbeater, V.A. Williams, Org. Process Res. Dev. 12 (2008) 41.

[15] F. Bergamelli, M. lannelli, J.A. Marafie, J.D. Moseley, Org. Process Res. Dev. 14 (2010) 926.

[16] C.R. Strauss, Org. Process Res. Dev. 13 (2009) 915.

[17] J.R. Schmink, C.M. Kormos, W.G. Devine, N.E. Leadbeater, Org. Process Res. Dev. 14 (2010) 205.

[18] H. Lehmann, L. LaVecchia, Org. Process Res. Dev. 14 (2010) 650.

[19] C. Leonelli, T.J. Mason, Chem. Eng. Process 49 (2010) 885.

[20] C.R. Strauss, R.S. Varma, Top. Curr. Chem. 266 (2006) 199.

[21] M.J. Gronnow, R.J. White, J.H. Clark, D.J. Macquarrie, Org. Process Res. Dev. 9 (2005) 516.

[22] J.D. Moseley, C.O. Kappe, Green Chem. 13 (2011) 794.

[23] M.Nüchter,B.Ondruschka,W.Bonrath, A. Gumb, Green Chem. 6 (2004) 128.

[24] J.D. Moseley, E.K. Woodman, Energy Fuels 23 (2009) 5438.

[25] T. Razzaq, C.O. Kappe, ChemSusChem 1 (2008) 123.

[26] C.O. Kappe, Chem. Soc. Rev. 37 (2008) 1127.

[27] J.M. Kremsner, A. Stadler, C.O. Kappe, Top. Curr. Chem. 266 (2006) 233.

[28] H. Lehmann, L. LaVecchia, Org. Process Res. Dev. 14 (2010) 650.

[29] T.N. Glasnov, C.O. Kappe, Macromol. Rapid Commun. 28 (2007) 395.

[30] J. Robinson, S. Kingman, D. Irvine, P. Licence, A. Smith, G. Dimitrakis, D. Obermayer, C.O. Kappe Phys. Chem. Chem. Phys. 12 (2010) 4750.

[31] V.L.M. Silva, A.M.S. Silva, D.C.G.A. Pinto, J.A.S. Cavaleiro, T. Patonay, Synlett 15 (2004), 2717.

[32] A.M.G. Silva, B. Castro, M. Rangel, A.M.S. Silva, P. Brandão, V. Felix, J.A.S. Cavaleiro, Synlett 6 (2009) 1009.

[33] V.L.M. Silva, A.M.S. Silva, D.C.G.A. 
Pinto, J. Elguero, J.A.S. Cavaleiro Eur. J. Org. Chem. (2009) 4468.

[34] S.M.M.Lopes, C.M.Nunes, T.M.V.D.Pinho e Melo, Tetrahedron 66 (2010) 6078.

[35] M. Pineiro, T.M.V.D. Pinho e Melo, Eur. J. Org. Chem. (2009), 5287.

[36] P.J. Figiel, M.N. Kopylovich, J. Lasri, M.F.C. Guedes da Silva, J.J.R. Fraús- to da Silva, A.J.L. Pombeiro, Chem. Commun. 46 (2010) 2766.

[37] M. Abrantes, P. Neves, M.M. Antunes, S. Gago, F.A.A. Paz, A.E. Rodrigues, M. Pillinger, I.S. Gonçalves, C.M. Silva, A.A. Valente, J. Mol. Catalysis A: Chemical 320 (2010) 19.

[38] T.R. Amarante, P. Neves, A.C. Coel- ho, S. Gago, A.A. Valente, F.A.A. Paz, M. Pillinger, I.S. Gonçalves, Organometallics 29 (2010) 883.

[39] P. Silva, A.A. Valente, J. Rocha, F.A.A. Paz, Cryst. Growth Des. 10 (2010) 2025.

[40] J. Klinowski, F.A.A. Paz, P. Silva, J. Rocha, Dalton Trans. 40 (2011) 321.

Atualidade Cientíica

\section{Nanopartículas Híbridas de Cobre-Ouro para Conversão de $\mathrm{CO}_{2}$}

O cobre é um dos poucos metais capazes de converter o dióxido de carbono em hidrocarbonetos com exigências energéticas relativamente baixas. Quando aplicado a um elétrodo e estimulado com a respetiva carga elétrica, o cobre atua como um catalisador, promovendo uma reação eletroquímica que reduz o $\mathrm{CO}_{2}$ a metano ou metanol. Deste modo, numerosos investigadores têm estudado o potencial do cobre como um meio energeticamente eficiente para reciclar as emissões de $\mathrm{CO}_{2}$ em centrais térmicas, permitindo a sua recuperação e posterior conversão em metano, que por sua vez poderia ser utilizado como fonte energética da própria central.

Este sistema possibilitaria uma redução significativa das emissões de gases com efeito de estufa em centrais térmicas de carvão e gás natural. No entanto, o cobre é um metal instável (que oxida facilmente), o que pode afetar negativamente as suas propriedades como catalisador, provocando um retardamento da reação e a produção de produtos secundários indesejados, como monóxido de carbono ou ácido fórmico.

Agora, investigadores do Massachusets Institute of Technology (MIT) propõem uma nova solução, que pode reduzir as necessidades energéticas na conversão de $\mathrm{CO}_{2}$ mediada por cobre, para além de possibilitar uma muito melhor estabilização do metal. O grupo concebeu nanopartículas de cobre combinado com ouro, que se revelam resistentes à oxidação. Deste modo, os investigadores verificaram que bastava adicionar uma pequena quantidade de ouro para tornar o cobre muito mais estável. Posteriormente demonstraram experimentalmente que elétrodos revestidos com as nanopartículas híbridas necessitavam de consideravelmente menos energia para converter $\mathrm{CO}_{2}$ do que os elétrodos revestidos com nanopartículas de cobre puro.

Um artigo detalhando os resultados será publicado no Chemical Communications. Assim, a coautora Kimberly Hamad-Schifferli, Professora Associada de Engenharia Mecânica e Engenharia Biológica no MIT, afirma "normalmente introduz-se bastante energia para se converter o dióxido de carbono em algo útil e agora demonstrámos que as nanopartículas de cobre-ouro são muito mais estáveis e revelam potencial para baixar as necessidades energéticas da reação".

A escolha de sintetizar o material à nanoescala deveu-se ao facto de "as partículas mais pequenas possibilitarem maiores áreas superficiais disponíveis para a interação com as moléculas de $\mathrm{CO}_{2}$ ".

Hamad-Schifferli trabalhou com Yang Shao-Horn, o Professor Associado Gail E. Kendall, de Engenharia Mecânica no MIT, o pós-doc Zhichuan Xu e Erica Lai. A equipa escolheu o ouro como um metal apropriado para combinar com o cobre, devido ao conhecimento prévio das suas propriedades, já que anteriormente se tinha verificado que esta combinação prevenia a oxidação do cobre. A síntese das nanopartículas foi realizada pela adição de sais contendo ouro a uma solução de sais de cobre. Esta solução foi aquecida para a produção de nanopartículas híbridas de cobre e ouro.

Posteriormente, Xu submeteu estas nanopartículas a uma sequência de operações, de forma a obter um pó que foi usado para revestir um pequeno elétrodo. Para testar a reatividade das nanopartículas, Xu introduziu o elétrodo na solução, onde borbulhou $\mathrm{CO}_{2}$. Depois aplicou uma reduzida diferença de potencial no elétrodo, e mediu a corrente resultante na solução. Supôs-se que esta corrente deveria ser um indicador fiável da eficiência da reação promovida pelas nanopartículas.

Deste modo, a equipa descobriu que o potencial aplicado para a obtenção de uma corrente estável era muito menor para as nanopartículas de cobre-ouro do que para as correspondentes de cobre ou de ouro puro, o que é um indicador de que a quantidade de energia requerida para manter a reação é muito menor no caso das nanopartículas híbridas em relação às nanopartículas de cobre puro. Hamad-Schifferli acrescenta que pretende observar com maior pormenor a estrutura das nanopartículas de cobre-ouro de modo a otimizar a sua configuração para a conversão de $\mathrm{CO}_{2}$.

No entanto, a investigadora reconhece que o revestimento de elétrodos à escala industrial com um material à base de ouro pode revelar-se dispendioso. Todavia, é possível que as poupanças de energia e o potencial de reutilização compensem os custos iniciais.

(adaptado do artigo de 11/04/2012 de Jennifer Chu: Hybrid Copper-Gold Nanoparticles Convert $\mathrm{CO}_{2}$, Chemical \& Engineering News - http://web.mit.edu/newsoffice/2012/hybrid-copper-gold-nanoparticles-convert-co2.html)

Paulo Brito (paulo@ipb.pt)

Instituto Politécnico de Bragança 